\title{
Facteurs d'humanités : accent et phrasé entre contrastes et programmes
}

Yves Citton

\section{(2) OpenEdition \\ 12 Journals}

Édition électronique

URL : http://journals.openedition.org/edl/835

DOI : $10.4000 /$ edl. 835

ISSN : 2296-5084

Éditeur

Université de Lausanne

\section{Édition imprimée}

Date de publication : 15 mai 2015

Pagination : 189-208

ISBN : 978-2-940331-45-1

ISSN : 0014-2026

\section{Référence électronique}

Yves Citton, "Facteurs d'humanités : accent et phrasé entre contrastes et programmes ", Études de lettres [En ligne], 1-2 | 2015, mis en ligne le 15 mai 2018, consulté le 19 décembre 2020. URL : http:// journals.openedition.org/edl/835; DOI : https://doi.org/10.4000/edl.835 


\section{FACTEURS D'HUMANITÉS: ACCENT ET PHRASÉ ENTRE CONTRASTES ET PROGRAMMES}

L'étude littéraire des textes est-elle porteuse de revendications éthiques, politiques, culturelles? A quelles échelles ces revendications émergent-elles au mieux? En quoi et envers quoi prenons-nous de la distance et de la hauteur en lisant des œuvres littéraires? Qu'apprenons-nous à faire (tout autant qu'à voir) en regardant les textes de très près? Cet article tente de répondre à ces questions en mobilisant la notion de "geste», et en défendant le principe d'un "droit aux Humanités», fondé sur notre besoin de reformuler ce que les formulaires et les programmes (éducatifs, bureaucratiques ou informatiques) ont pré-paramétré pour nous.

Comment valoriser aujourd'hui le travail effectué par un professeur de Lettres? Face à la baisse des effectifs inscrits dans certaines filières littéraires, il n'est pas superflu de se demander ce que nous faisons (de bien et de mal) en enseignant la littérature, comment et pour quoi nous le faisons. J'esquisserai quelques réponses à ces questions en suivant une échelle qui me fera d'abord regarder les textes de très loin, pour m'approcher progressivement des détails qui en font la richesse propre. Ce sera l'occasion de se demander quels gestes sont les plus appropriés à chaque échelle, pour rendre justice à la fois aux textes lus et aux formes de subjectivation qui s'en nourrissent.

\section{Faire des Humanités des agents de décloisonnement}

La première et sans doute la plus importante condition de survie, voire de reconquête, des études littéraires, dans le contexte peu favorable 
qu'elles traversent actuellement, me paraît être de décloisonner nos filières. Ce qui plombe sans espoir nos destins, c'est la spécialisation qui, dès le niveau de la licence, dans un pays comme la France, contraint des étudiants de littérature à ne faire "que» de la littérature, et qui empêche ou dissuade les étudiants de chimie ou d'économie d'étudier des textes littéraires ou philosophiques. Les Humanités n'auront d'avenir que si nous parvenons, avec nos collègues des autres disciplines, à casser ces cloisonnements étouffants. Il faut mettre les Humanités partout, sans quoi elles ne survivront nulle part.

Mais cela vaut aussi bien pour les autres disciplines: au moins jusqu'à la fin de la licence, mais aussi dans une part de leurs études de Master, les étudiants doivent être encouragés à se familiariser simultanément avec les recherches menées en sciences de la nature et en sciences sociales et en Humanités. Le niveau d'intellectualité requis par la complexité des problèmes que nous devons affronter en tant qu'électeurs, travailleurs, consommateurs, parents est tel que nos sociétés ne peuvent pas se permettre de ne pas nous donner une formation non seulement de base, mais supérieure (à savoir nous initiant aux procédures de la recherche), dans tous ces domaines à la fois.

De ce point de vue, loin d'être marginaux, nous autres Lettreux sommes au cœur de ce besoin impératif de décloisonnement des domaines de recherche et d'enseignement. Les Humanités peuvent en effet apparaitre comme fournissant des plates-formes interdisciplinaires (ou plutôt indisciplinaires) facilitant la circulation et les regards croisés des disciplines entre elles: peut-on lire un roman sans se faire un peu historien, un peu linguiste, un peu philosophe, un peu psychologue, un peu théoricien de la politique? La pratique des Humanités est, en elle-même, un facteur de décloisonnement et de mise en dialogue des disciplines.

\section{Revendiquer une expérience d'exaltation}

A cette échelle, qui est la plus large possible, les textes que nous étudions sont donc à revendiquer comme des lieux de mise en perspective réciproque des savoirs qui circulent au sein d'une société - savoirs d'hier, relatifs aux textes du passé que nous lisons avec nos étudiants, et savoirs d'aujourd'hui, relatifs aux enjeux, aux pertinences et aux significations que nous y reconnaissons en l'interprétant ensemble. L'échelle 
macroscopique des textes, ce serait donc celle qui les réinscrit dans le tissu des débats sociaux qui ont présidé à leur émergence passée et/ou qui conditionnent leur réception présente. A ce niveau nécessairement surplombant, le texte vit en tant qu'on peut se l'approprier, l'investir de pertinences actuelles, l'utiliser dans des conflits en cours - ce qui implique forcément de prendre le risque de le détourner de ses coordonnées et de ses visées initiales. C'est peut-être parce que les étudiants des trente dernières années nous ont insuffisamment vus investir les textes passés avec l'expérience de nos vies présentes que nos salles de classe ont partiellement perdu de leur attractivité ${ }^{1}$.

Cet investissement ne se borne bien entendu pas à des appropriations politiques, activistes ou propagandistes, comme cela a été le cas outreAtlantique avec les combats menés par les minorités raciales, les féministes ou les mouvements queer, pour revendiquer des droits sociaux à travers des recherches et des enseignements relevant des Humanités. Si cela constitue un modèle possible, qui a tout à la fois ses vertus et ses limites propres, le décloisonnement et l'appropriation des textes peuvent passer aussi bien par la passion qu'on investit dans la lecture de dictionnaires ou dans l'étude de chansons. L'enjeu principal me paraît être de revendiquer pour nos études un élan d'amour et d'exaltation, qui ne paraît incompatible avec les exigences de scientificité associées à l'éthos universitaire que dans une vue étroite, asséchante, irréaliste et mutilante de ce que sont la science et l'université.

\section{Promouvoir une étude pratique des gestes}

En descendant d'un cran dans l'échelle, pour nous situer au niveau intermédiaire de notre traitement des textes, j'aimerais mettre au premier plan la notion de "geste», qui me semble à situer au cœur de nos expériences esthétiques. Les psychologues nous apprennent en effet que c'est par l'entremise des gestes que nous nous approprions cognitivement les différents éléments de notre environnement. Les concepts (résultats de notre capacité à concipere) sont ce par quoi nous parvenons à "saisir"

I. Pour une bonne réflexion, inspirée de Bruno Latour, sur cette question, voir R. Felski, "Context Stinks!». Pour des développements plus techniques, je renvoie à mon ouvrage Lire, interpréter, actualiser. Pourquoi les études littéraires? 
des liens de causalité. Nos rapports aux autres humains sont affaire de "postures», bien davantage que d'actes. Nos comportements ont le statut de gestes dès que nous les savons être signifiants, visibles et interprétables par autrui - à savoir presque toujours.

Or la nature du geste se présente à nous comme un nœud de contradictions. Les mouvements que j'identifie comme «mes" gestes ne font le plus souvent que me traverser, puisque c'est généralement par imitation d'autrui que je les ai appris. En même temps que nos gestes peuvent représenter le sommet de notre capacité de maîtrise de soi, par exemple lors d'une audition de conservatoire de musique, les gestes qui nous exaltent le plus sont ceux qui échappent partiellement à notre contrôle: un geste «technique» n'emporte notre admiration que s'il s'expose à un risque d'échec, en essayant de dépasser sa simple technicité. Par contraste avec un "acte", censé relever de la réalité, un geste s'inscrit dans la sphère de la représentation et du spectacle: faire le geste de frapper ne produit que l'apparence d'un coup. Et pourtant, nos gestes nous collent à la peau: faire le geste de frapper quelqu'un constitue un acte en soi, qu'on peut nous reprocher à l'occasion. Bref, tout ce que nous faisons relève, d'une manière ou d'une autre, du geste, et pourtant ce n'est jamais vraiment, ou jamais seulement, nous qui agissons à travers nos gestes. Ou plutôt oui : c'est toujours «nous», et jamais «moi», qui est l'auteur du geste que j'accomplis.

Si les Humanités ont une fonction primordiale, c'est de nous permettre de réfléchir sur les gestes qui nous traversent. Lire un roman de l'abbé Prévost, voir un tableau du Caravage, un film d'Hitchcock ou une série télévisée, c'est se plonger dans une immersion narrative où, par empathie, nous sommes conduits à accomplir mentalement les gestes relationnels opérés par les protagonistes. Dans la mesure où nos études redoublent cette expérience d'immersion par une prise de distance critique, les Humanités nous apprennent à vivre d'une façon réfléchie les gestes qui nous traversent ${ }^{2}$.

2. Une telle formulation du travail interprétatif induit par la pratique et l'étude de la littérature exprime à travers la notion de «geste» ce que Claude Reichler, dans «La littérature comme interprétation symbolique», exprimait à travers celle de «modélisation" - lorsqu'il affirmait par exemple que "les textes qu'on appelle littéraires sont des interprétations symboliques non collectives, dont la fonction absolument spécifique consiste à permettre à un sujet d'interpréter par le langage les modélisations elles-mêmes» (p. 111). Nos gestualités esthétiques sont des interprétations singularisantes, dont la 
La littérature, du moins telle que nous la concevons depuis un peu plus de deux siècles, peut donc être envisagée comme une vaste machine d'inculcation de gestes relationnels, machine largement relayée depuis 1900 par le cinéma, puis par la télévision. Si nous nous comportons comme nous le faisons avec nos semblables et notre environnement, c'est que nous avons baigné dans cette inculcation depuis notre plus tendre enfance, d'abord en grandissant parmi des tribus de lecteurs et de spectateurs, puis en devenant lecteurs et spectateurs nous-mêmes. C'est non seulement la littérature, et avec elle le théâtre, le cinéma, la télévision, qui sont à concevoir comme une fabrique collective de nos gestes individuels, mais la pratique des Humanités elle-même.

A l'horizon de la revalorisation de nos (in)disciplines, nous serions bien avisés de réévaluer la notion d' "exemplification", fondée sur le pouvoir de contagion rayonnante dont sont porteurs nos gestes, notion récemment revisitée par plusieurs philosophes et théoriciens de la littérature $^{3}$. Je citerai l'un d'eux, Daniele Giglioli, qui décrit en ces mots les conditions de survie de la critique et de la théorie littéraires:

D'interprétation, elles doivent se faire exemplification, se penser, davantage que comme pensée et communication, plutôt comme geste, performance, événement, processus constituant qui se donne ses règles dans l'acte qui le fait advenir: paradigme, modèle, exemple d'une politique des vérités (pour paraphraser Badiou) que l'on ne peut atteindre qu'à travers cet exemple même. Ce n'est pas tant ce que je dis, ni la méthode avec laquelle je le dis, qui comptent, mais plutôt le fait que les techniques que j'emploie me permettent de prendre efficacement la parole. Ne regardez pas ce qu'il y a dans le texte, regardez ce qu'il est possible de faire en lisant ce texte ${ }^{4}$.

Autant que d'étudier nos gestes de façon réfléchie, les Humanités nous invitent donc à "pratiquer» - avec les textes - certains gestes dont c'est la vertu contagieuse, autant que réflexive, qui fait le principal mérite.

fonction consiste à permettre à un sujet d'interpréter réflexivement les gestes qui le traversent et le constituent.

3. Voir par exemple, à vingt ans de distance, Ch. Kelly, Rousseau's exemplary life, T. Hampton, Writing from History, J. Goma Lanzon, Ejemplaridad publica.

4. D. Giglioli, «Trois cercles», p. 64. 


\section{Orienter nos subjectivations}

La dynamique commune au geste et à l'expérience esthétique me paraît être celle d'une individuation permettant de se réapproprier des comportements imitatifs qui ne font originellement que nous traverser, et que nous ne pouvons faire nôtres qu'en leur apportant une inflexion personnalisante. Comme le signale très clairement Daniele Giglioli, l'enjeu des études littéraires est d'aider chacun à orienter ses processus de subjectivation:

Comment être mieux formé à faire et à défaire un sujet que par ce que nous fournit l'usage exemplaire que nous faisons des textes littéraires? L'exemplification est la matrice de toute subjectivité possible à venir. [...] La littérature ne représente jamais un "qu'est-ce que»: elle dit «qui ». Elle met en scène un agent, une conscience, un réseau de rapports entre des consciences possibles. Elle présuppose un sujet parce qu'elle l'imagine. Elle rend familier l'usage de soi à travers l'autres

$\mathrm{Si}$, à l'échelle la plus large, notre pratique des textes nous aide à cartographier la circulation conflictuelle des discours, des idées, des représentations qui structurent notre époque, à l'échelle intermédiaire des gestes, les Humanités nous aident à "former» une subjectivité capable de soutenir les tensions contradictoires qui nous tiraillent, et parfois nous déchirent, au sein de notre tissu relationnel. Le geste propre aux études littéraires - l'explication de texte - consiste à analyser, mesurer, peser, évaluer des gestes relationnels que la lecture rapprochée nous permet d'observer au ralenti. Nous formons nos subjectivités en train de se faire en réfléchissant sur des gestes en train de s'accomplir. Nous rendons "publique» l'exemplarité contenue dans chacun de nos comportements sociaux. Nos compassions ou nos détestations - réfléchies parce que ralenties - envers les gestes que nous observons nous aident à nous orienter parmi les multiples réactions possibles à certains types de situation. Ce sont nos goûts et nos dégoûts que nous mettons à l'épreuve de l'interprétation des textes, et que nous reconfigurons en fonction de ce que ces textes nous aident à sentir.

On voit ainsi apparaître l'une des fonctions sociales permettant de valoriser aujourd'hui le travail effectué par un professeur de Lettres:

5. Ibid., p. 65. 
nous aider à surmonter la schizophrénie de plus en plus inhérente à nos modes de subjectivation tendus entre des exigences de moins en moins compatibles entre elles. Pendant longtemps, dans nos contrées, ce sont les prêtres qui, le dimanche matin, interprétaient des textes (sacrés) pour aider les fidèles à donner sens aux accidents et aux difficultés de leur existence. Depuis un siècle, ce sont parfois des psychanalystes qui aident certains d'entre nous à le faire, à grands frais. A travers des livres et des cours, en interprétant des textes sacralisés par deux siècles d'érudition philologique et de rituels exégétiques, les études de Lettres constituent un des lieux de "facilitation" de ces processus de subjectivation non schizophrénique - et les faibles financements qu'on leur accorde sont peut-être l'un de nos investissements les plus "économiques", dès lors qu'on mesure les coûts induits par les explosions de schizophrénie individuelle ou collective. Comme le dit le slogan américain: If you think education is expensive, try ignorance! Si vous trouvez que les Humanités coûtent cher, attendez de voir le coût de la schizophrénie...

\section{Développer une science des nuances}

Ce que je viens d'esquisser au niveau de cette échelle intermédiaire que sont les gestes aura sans doute paru naïf, voire rétrograde. Lire des romans, est-ce forcément s'identifier à un protagoniste, singer dans nos têtes les mouvements accomplis dans la fiction - comme si l'expérience littéraire se confondait avec une partie de Wii? Bien sûr que non. Autant qu'une plate-forme d'interaction entre discours, autant qu'une expérience immersive inculcatrice de gestes, les Humanités nous fournissent, à l'échelle microscopique de notre pratique des textes, un espace de sensibilisation stylistique ${ }^{6}$. Par comparaison avec la noble tâche d'enrayer notre schizophrénie prévalente, ce travail de sensibilisation stylistique pourra paraître anodin, quoique sans doute plus proche de l'idée commune que nous nous faisons de notre profession. J'aimerais pourtant montrer que c'est sur lui que reposent finalement les autres fonctions qu'on peut reconnaître aux Humanités.

La véritable spécificité de notre travail se définit par une question d'échelle et de tempo. Dans un monde idolâtrant le global, la vitesse et

6. Voir à ce propos le beau livre de M. Macé, Façons de lire, manières d'être. 
l'accélération, les Lettreux doivent revendiquer la nécessité de la lenteur et la puissance de l'infinitésimal. Suspendre la course hebdomadaire au profit, à l'augmentation, à la croissance, à la connaissance et à l'innovation, pour passer une heure à lire, relire, étudier, analyser, discuter et interpréter quelques lignes d'un texte vieux de plusieurs décennies ou de plusieurs siècles et qui, en tant que fiction, ne nous "apprend" de toute façon rien de "vrai» ou d'immédiatement "utile» sur notre réalité - voilà en effet qui paraît condamner l'enseignement littéraire à relever de la perte de temps, si ce n'est de la recherche de temps perdu. C'est pourtant quand nous nous perdons dans le détail d'un texte que nous nous livrons à l'activité non seulement la plus «valorisable», mais la plus fondamentalement "valorisante». Reprenons brièvement chaque terme de ce paradoxe.

Un texte, pour un esprit littéraire, c'est d'abord une occasion de «se perdre». Si nous nous y retrouvions à la première lecture, nous n'aurions pas besoin de l'interpréter. Tout notre travail vise moins à comprendre et à expliquer des textes qu'à chercher des points où nous ne le comprenons pas, des points de résistance, de non-savoir, de non-sens, d'affolement de nos boussoles, où, à travers cette expérience de perte de sens, nous pouvons espérer contribuer à l'émergence de sens nouveaux, de compréhensions renouvelées, élargies, ajustées, affinées - et participer ainsi à la constitution de nouvelles valeurs. Il faut ici commencer par inverser les attentes et les rôles sur lesquels repose le ronronnement universitaire: toute heure où l'enseignant n'a pas dû avouer son ignorance, son insuffisance et son incapacité à comprendre et à expliquer un texte est une heure perdue pour la science littéraire!

Quel est l'objet propre de cette "science" qui commence par nous perdre dans le désarroi et la désorientation? Le détail, ou plus précisément "la nuance», telle que la définissait Roland Barthes, qui rêvait, à la fin de sa vie, de recomposer les études littéraires en «sciences de la nuance» ou «diaphoralogie»:

La pratique (générale: mentale, écrite, vécue) de l'individuation, c'est la Nuance [...] : la prendre fortement, généralement, théoriquement, pour une langue autonome; à preuve qu'elle est névrotiquement censurée, refoulée par la civilisation grégaire d'aujourd'hui. On peut dire que la civilisation des médias se définit par le rejet (agressif) de la nuance. [...] Comme Différence, la Nuance est sans cesse en contraste, 
en bataille contre ce qui l'entoure, l'opprime, ce dont elle cherche par un sursaut vital à se distinguer ${ }^{7}$.

On est ici à l'articulation de deux échelles: la science de la nuance, à l'échelle microscopique du détail, conditionne la pratique de l'individuation, à savoir de la subjectivation qui caractérisait l'échelle intermédiaire du geste. On ne peut espérer surmonter notre schizophrénie native et cultiver notre singularité qu'en prêtant attention aux nuances des mots, des attitudes, des gestes, des apparences qui nous entourent et qui nous constituent. Pourquoi le texte emploie-t-il ce terme-là, plutôt qu'un autre qu'on dit être son synonyme? Pourquoi ce contraste de ton? Pourquoi cet accent? Pourquoi ce phrasé?

\section{L'accent et le phrasé}

On reconnaît là des questions qui ont traversé l'ensemble des réflexions, des écrits et des enseignements auxquels s'est consacré André Wyss, depuis son rôle public de Monsieur Dictionnaire à la Télévision Suisse Romande et ses enseignements d'éloquence dissertative à l'Université de Genève, jusqu'à ses cours lausannois sur la poésie et à ses livres sur l'accent chez Rousseau et sur L'Eloge du phrasé. Dans ce dernier ouvrage, il écrit que «le phrasé [est] l'équivalent dans la musique de ce qu'est l'inflexion dans la parole et le geste dans l'action». Le propre du geste, de la parole ou de toute forme non seulement d'art, mais plus largement de vie - ce qui la singularise -, ce sont justement des nuances: phrasés, inflexions, accents.

Comme le phrasé, l'inflexion est constituée de différences ténues et non mesurables de dynamique, de rythme, de timbre aussi. Comme le phrasé, le geste est entre l'exécution (d'une action) et l'expression (d'un état d'âme); comme lui, il est plus ou moins volontaire, n'est jamais rigide, doit renoncer à toute explication ${ }^{8}$.

Le phrasé de l'instrumentiste, le plié du danseur, l'accent de la parole, le coup de pinceau du peintre, le doigté de l'amant: autant de gestes

7. R. Barthes, La préparation du roman, séance du 27 janvier 1979, p. 81-83.

8. A. Wyss, Eloge du phrasé, p. 280. 
qui nous subjectivent et nous individuent par le travail propre de leurs nuances. De telles réalités neutralisent l'apparente contradiction entre style et substance. De par leur double nature d'«exécution (d'une action)" et d'"expression (d'un état d'âme)", ces gestes appellent à un renversement permanent de l'extérieur dans l'intérieur, de l'intime dans le manifeste, et surtout d'une position de maîtrise (impliquée par «l'exécution" d'une action) dans une attitude d'accueil réceptif de l'étrangeté, où le sujet se constitue grâce à un mouvement d' "expression " qui le traverse - de façon toujours "plus ou moins volontaire", "jamais rigide", toujours revêche à l'« explication». Ce nouage paradoxal d'exécution maîtrisée et de réceptivité expressive travaille l'une des citations de Rousseau qui sert de pivot au livre d'André Wyss sur l'accent ${ }^{9}$ (lequel désigne ici les inflexions de la voix):

Les passions ont leurs gestes, mais elles ont aussi leurs accents, et ces accents qui nous font tressaillir, ces accents auxquels on ne peut dérober son organe, pénètrent par lui jusqu'au fond du cœur, y portent malgré nous les mouvements qui les arrachent, et nous font sentir ce que nous entendons ${ }^{10}$.

C'est par le jeu des nuances que nous habitons nos gestes: ce sont elles qui «nous font tressaillir», qui "pénètrent jusqu'au fond du cœur», qui nous "arrachent nos mouvements" les plus significatifs, et qui nous font «sentir» ce que nous percevons. Ces "détails» que sont l'accent, le phrasé, l'inflexion constituent en réalité les lieux privilégiés de notre subjectivation, en tant que celle-ci est toujours passionnée - au triple sens de "subie» ("assujettie» aux flux d'affects sociaux qui se tissent collectivement à travers nous), d'" énergisée" (par l'emportement de ces affects même) et de "personnalisée» (puisque n'y prend pas forme un savoir à prétention universalisante, mais un positionnement "subjectif»).

C'est donc autant sur un culte - au sens de "culture» - que sur une «science» de la nuance que reposent nos études de Lettres. Se perdre dans les détails d'un texte est productif, non pas en ce que cela fabrique des objets précieux, mais en ce que cela cultive des sujets mieux capables d'évaluer non seulement le prix de toute chose mais aussi la dignité d'autres sujets. Toute l'entreprise littéraire me paraît à concevoir comme

9. A. Wyss, Jean-Jacques Rousseau. L'accent de l'écriture.

Io. J.-J. Rousseau, Discours sur l'origine des langues, p. 63. 
un tel culte, avec ce que cela implique à la fois de travail de cultivation et de rituel de sacralisation.

\section{Le droit aux Humanités}

Le culte des nuances doit en effet être sacralisé parce que, comme le soulignait très pertinemment Roland Barthes voilà trente ans déjà, il est «en bataille" contre "la civilisation des médias", "qui l'entoure, l'opprime" et qui "se définit par le rejet (agressif) de la nuance». Le "sursaut vital» par lequel la nuance «cherche à se distinguer» du grégarisme médiatique relève d'un besoin de singularisation qui anime chacun de nous - et dont se nourrit le renouvellement constant des formes et des contenus médiatiques eux-mêmes. La sacralisation ou, plus précisément peut-être, la "sanctuarisation" des lieux de culture des nuances, tels que ceux que l'on regroupe sous la bannière des Humanités, est donc à concevoir à la fois comme une bataille contre certaines tendances du monde actuel et comme un combat interne à la dynamique propre à ce monde actuel lui-même.

Comme le montrent abondamment les ouvrages de Bernard Stiegler ${ }^{11}$ et de nombreux autres analystes du contemporain, une certaine forme de consommation et de communication de masse est en train d'écraser les énergies dont se nourrit notre méga-machine médiatico-consumériste. Ce qui est menacé d'épuisement, ce sont non seulement les énergies physico-chimiques que nous extrayons de la planète, mais aussi les énergies «libidinales» qui alimentent la production et la demande des biens et des services que nous échangeons. A partir de telles analyses, on peut construire un argumentaire assez crédible pour montrer que non seulement notre "culture", mais le capitalisme lui-même ont besoin des Humanités, s'ils entendent pouvoir persévérer dans leur être.

Le problème est que la logique capitaliste actuellement dominante semble de moins en moins capable d'entendre raison, et de se réorienter vers ce qui pourrait assurer non seulement «sa» mais «notre" survie collective. C'est donc contre son emballement suicidaire qu'il me semble aujourd'hui nécessaire de batailler (pour les nuances) en revendiquant

II. Voir, entre autres, B. Stiegler, Economie de l'hypermatériel et psychopouvoir et B. Stiegler et al., Réenchanter le monde. 
un «droit aux Humanités». La référence aux «humanités» permet en effet de faire coïncider, d'une part, une revendication de politique culturelle, le financement d'un certain nombre de disciplines jadis placées au cœur de nos dispositifs pédagogiques élitistes et aujourd'hui reléguées à une position de plus en plus marginale dans nos institutions éducatives démocratisées, et, d'autre part, une revendication géopolitique, la promotion des différences culturelles qui font la richesse commune de nos multiples formes d'humanités, telles qu'elles se sont déployées aux quatre coins de la planète, à travers des phases d'échanges, de conflits et de redimensionnements incessants ${ }^{12}$. Or certaines tendances de la logique actuelle du capitalisme menacent simultanément ces deux acceptions de la référence aux humanités.

\section{L'entrejeu des programmes et des contrastes}

J'aimerais caractériser très grossièrement la situation présente par un grippage de la dynamique qui a prévalu jusqu'ici entre programmes et contrastes. Ce terme de "contraste», que Barthes utilise dans la citation évoquée ci-dessus, resitue la nuance dans le cadre ontologique proposé par le philosophe Alfred North Whitehead (1861-1947) ${ }^{13}$. Celui-ci décrit notre univers comme offrant à nos sens, à nos connaissances et à nos pratiques une infinité de contrastes et de devenirs en évolution permanente. Il rend compte de notre capacité à nous orienter dans ce monde fluide en décrivant des processus de "concrescence», par lesquels certains contrastes prennent une saillance qui nous permet d'y reconnaître des objets distincts et dotés d'une certaine stabilité. Sa philosophie nous invite donc à envisager que tout est fait de contrastes et que pourtant certaines nuances gagnent par concrescence une forme d'existence capable de leur donner une "substance» propre.

Comme toute forme de vie individuelle ou collective, le capitalisme se développe en exploitant la diversité des nuances que lui fournissent les contrastes dont se compose notre réalité. Comme toute forme d'organisation sociale, il s'efforce de maîtriser les processus de concrescences

I2. Pour une réflexion plus étendue sur les implications de cette coïncidence autour des différents sens des humanités, je renvoie à L'avenir des humanités.

13. Voir A. N. Whitehead, Procès et réalité. 
en les dirigeant par des activités qu'on peut globalement faire relever de la programmation, entendue ici dans son sens étymologique le plus large d'«écrit à l'avance». Toute forme de projet (industriel, architectural, artistique) relève donc d'une activité de programmation, en ce sens que son agent essaie de prescrire quel devra être son déroulement et ses résultats.

L'industrialisation progressivement imposée à l'ensemble de la planète depuis le début du XIX ${ }^{\mathrm{e}}$ siècle, relayée et exacerbée par l'informatisation à laquelle nous sommes en train d'assister actuellement, modifie toutefois profondément les modalités et les effets de cette activité de programmation constitutive de notre humanité. De même que les programmes machiniques de l'usine ont accéléré, homogénéisé, uniformisé et finalement détruit les gestes corporels développés par des générations d'artisans au fil des siècles antérieurs, de même les logiciels de nos ordinateurs tendent-ils aujourd'hui à accélérer, homogénéiser, uniformiser, voire détruire un certain nombre d'opérations mentales développées parmi les humains au cours des périodes antérieures.

\section{Questions d'échelle, questions de survie}

Or ce qui se perd en passant du geste humain, qu'il soit corporel ou mental, à la machine, qu'elle soit chaîne de montage ou logiciel, ce sont les nuances et les contrastes par lesquels l'intelligence humaine "s'ajuste» incessamment aux nuances et contrastes qu'elle rencontre dans son environnement. En tant qu'il résulte de la collaboration des intelligences humaines, le capitalisme s'est nourri de part en part de cet ajustement réciproque des contrastes: c'est précisément cette capacité (proprement «libérale») d'ajustement aux nuances qui a assuré sa supériorité sur les rigidités de la programmation planifiée mise en place par les expériences de "socialisme réel». Mais ce à quoi on assiste aujourd'hui, à travers les crises financières et économiques bien entendu, mais beaucoup plus encore à l'occasion de la crise écologique vers laquelle nous nous dirigeons toutes voiles dehors, c'est à une emprise généralisée de la programmation, favorisée par sa capacité à produire plus rapidement de plus gros profits. Or cette emprise tend à paralyser de plus en plus 
dramatiquement notre capacité à ajuster nos nuances comportementales aux contrastes dont se compose la réalité ${ }^{14}$.

On le voit, tout ceci est précisément une question d' "échelle». La logique économico-financière nous étouffera parce qu'elle ne peut structurellement envisager que le trop court terme, rejetant la survie des générations ultérieures comme une nuance sans pertinence immédiate en termes de profits marchands. Ceux-ci reposent depuis l'émergence du capitalisme industriel sur des économies d'échelle: on gagne plus en produisant davantage de marchandises de façon plus uniforme. Le renversement du "fordisme» en "toyotisme», qui permet désormais la production en flux tendus de marchandises finement ajustées aux préférences du client, n'assouplit le fonctionnement des machines d'usine qu'en généralisant la programmation par logiciel, et donc en machinisant nos esprits.

Ce qu'il faut sanctuariser, par et pour la survie des humanités, c'est donc le "tout petit" (le détail, la nuance, le contraste à peine perceptible), en tant que ce tout petit, dans sa concrétude, contient la réserve de nouvelles concrescences grâce auxquelles se reconstituent, se renouvellent et se réorientent sans cesse nos pratiques proprement humaines. Se perdre dans le détail d'un texte, c'est faire l'expérience de ce «tout petit", de cette échelle la plus infime qui, dans ses accents, ses inflexions, ses phrasés, nous permet de ré-envisager sous une lumière nouvelle les implications cachées de nos programmations omniprésentes. Cette attention au tout petit apparait comme un «luxe», du point de vue d'une logique économico-financière qui ne valorise que le court terme des gros profits. C'est toutefois à ce luxe, dont vivent les Lettres, qu'est suspendu l'avenir de nos humanités.

\section{Pour un droit à la reformulation}

Je conclurai cette réflexion bien trop rapide et bien trop sommaire - qui contredit caricaturalement l'éloge de la lenteur et du détail qu'elle prétend promouvoir - en esquissant trois revendications directement issues de l'échelle microscopique à laquelle nous envisageons les textes dans nos salles de classe. Ces trois revendications textualistes me semblent en effet

I4. Voir sur ce point B. Hibou, La bureaucratisation du monde à l'ère néolibérale. 
pouvoir être généralisées de façon à recevoir une portée politique, voire géopolitique, immédiate et urgente ${ }^{15}$.

Face à l'emprise croissante des programmes sur nos vies, la défense des humanités passe d'abord par la revendication d'un droit à la reformulation. Nos nouvelles machines saturent notre monde de "formulaires" - à savoir de formes d'interactions préparamétrées qui conduisent nos conduites en ne nous laissant le choix qu'entre des alternatives déjà choisies pour nous. La pratique de l'explication de texte, et plus largement de la critique littéraire, constitue l'antithèse du formulaire, en ce qu'elle nous invite à questionner et à reconfigurer les formulations préexistantes pour en déplacer le cadrage, pour en accentuer les nuances, pour en marquer les contrastes inaperçus, de façon à en renouveler la signification. Critiques littéraires, historiens de l'art, philosophes, mais aussi sociologues et anthropologues passent leur temps à reformuler ce qui a déjà été formulé par autrui. Plus généralement, nos gestes ne sont humains (irréductibles à des programmations machinisées), et ne nous donnent l'occasion de concrétiser nos subjectivations, que dans la mesure où nous parvenons à leur insuffler une nuance qui nous soit propre, et qui en fasse émerger un contraste singularisant.

\section{Pour un droit à l'opacité}

Une deuxième revendication touche à ce qui nourrit le besoin constant de réinterprétation et de reformulation: la reconnaissance d'un problème au cœur de toute communication autre que purement fonctionnelle. Comme on l'a vu plus haut, il n'y a rien à interpréter pour qui croit tout comprendre ${ }^{16}$. Il n'y a rien à reformuler si les formulations existantes nous apparaissent comme transparentes. On comprend dès lors que la défense des humanités ait conduit Edouard Glissant à « réclam[er] pour tous le droit à l'opacité»: "Le consentement général aux opacités particulières est le plus simple équivalent de la non-barbarie.» ${ }^{17}$

I5. Ces derniers paragraphes suivent de près la conclusion de mon ouvrage Gestes d'humanités.

I6. Voir sur ce point R. Shusterman, Sous l'interprétation.

I7. E. Glissant, Poétique de la relation, p. 209 (je souligne). Pour une excellente présentation synthétique de la pensée archipélique de cet auteur important, voir le livre d’A. Ménil, Les voies de la créolisation. 
$\mathrm{Au}$ fur et à mesure que les instances de programmation se mondialisent, avec les risques de standardisation et de centralisation qu'impliquent les nouvelles formes de transparence permises par nos nouvelles machines, protéger des réserves d'implicite devient une condition de survie de la diversité culturelle. Les humanités n'alimentent leurs dynamiques réciproques que dans la mesure où elles sont une source d'interrogation et d'incompréhension les unes pour les autres. Là où le monde des programmes et des formulaires, des statistiques et des sondages, des réalités augmentées et de l'inter-communicabilité généralisée, projette l'utopie d'une transparence intégrale, le culte de la nuance qui définit l'échelle microscopique des études littéraires est indispensable pour entretenir les ressources de sens propres à l'opacité.

\section{Pour un droit à l'équivoque}

Non moins que sur l'opacité, qui nous donne l'impression subjective d'un manque de sens, les humanités reposent sur la dynamique propre à «l'équivoque», qui nous donne au contraire l'impression d'un excès de sens possibles et ambigus. Au lieu de réduire l'équivoque à "l'une des nombreuses pathologies qui menacent la communication", l'anthropologue brésilien Eduardo Viveiros de Castro propose d'en faire «une catégorie proprement transcendantale, une dimension constitutive du projet de traduction culturelle propre à $[s]$ a discipline»: "L'erreur ou l'illusion par excellence consisterait, justement, à imaginer qu'il y ait un univoque sous chaque équivoque, et que l'anthropologue en serait le ventriloque. ${ }^{18}$

Telle est bien une forme de barbarie propre à notre modernité programmatrice: espérer rendre le monde parfaitement univoque, en résorbant à la fois toute opacité derrière laquelle un sens serait caché, et toute équivoque à l'occasion de laquelle le sens fuirait en des directions contradictoires entre elles. Revendiquer "un droit à l'équivoque", c'est travailler à maintenir ouvert un espace propre à l'interprétation - en tant que celle-ci n'est pas résorption de la signification à un sens préconçu, mais émergence de sens nouveaux, qui se trament déjà dans le tissu de

I8. E. Viveiros de Castro, Métaphysiques cannibales, p. 56 et 59. 
nos pertinences entrecroisées, mais que nos systèmes de signification échouent encore à prendre en charge.

Nos entreprises de programmation s'efforcent - parfois pour de très bonnes raisons - de «traduire» nos gestes et nos comportements en fonctions algorithmiques calculables par des machines. Comme la traduction linguistique, qui nous permet de communiquer à travers nos différences d'origines, ce travail mérite d'être sans cesse raffiné, de manière à minimiser la violence qu'il impose aux sens de nos pratiques. En même temps que nous devons "raffiner nos traductions", notre culte des contrastes nous donne aussi l'occasion de "cultiver des intraduisibles", dont les opacités et les équivoques constituent des réserves de sens indispensables à la poursuite de l'aventure humaine. Traduire, en effet, ce n'est pas abolir l'intraduisible, mais lui ouvrir de nouveaux espaces en le repoussant plus loin: comme l'écrit encore Eduardo Viveiros de Castro, «traduire, c'est s'installer dans l'espace de l'équivoque et l'habiter. Non pas pour le défaire, car cela supposerait qu'il n’a jamais existé, mais, bien au contraire, pour le mettre en valeur ou le potentialiser» ${ }^{19}$.

\section{Facteurs d'humanités}

Droit à la reformulation, droit à l'opacité, droit à l'équivoque : c'est par l'exercice actif et très concret de ces trois formes de droit que le travail effectué par des professeurs de Lettres contribue aujourd'hui à humaniser nos sociétés industrialisées en voie rapide d'informatisation. Qu'ils commencent à prendre leur fonction ou qu'ils la quittent, ces professeurs travaillent toujours à partir d'une position de «retraite» par rapport aux flux constants de la communication. Nos salles de classe comme nos bibliothèques doivent offrir l'espace de vacuoles protégées de ces flux, où c'est parce que nous avons débranché nos téléphones portables et nos messageries électroniques que nous pouvons nous offrir le luxe de prêter attention aux accents autant qu'aux slogans, aux phrasés autant qu'aux contenus, aux gestes autant qu'aux actions.

19. Ibid., p. 57. Sur ces questions, voir aussi la belle présentation de B. Cassin au Vocabulaire européen des philosophies, l'essai d'E. Apter, The translation zone, que présente bien son entretien "Pour une politique de la traduction" paru dans la Revue des Livres, ainsi que le livre récent d'A. Renken, Babel heureuse - Pour lire la traduction. 
Que ce soit à l'échelle macroscopique du dialogue entre les disciplines, à l'échelle individualisée de nos subjectivations gestuelles ou à l'échelle microscopique des nuances et des contrastes, les professeurs de littérature apparaissent comme des «facteurs d'humanités». En aidant les Lettres d'hier à rencontrer de nouveaux destinataires aujourd'hui et demain, ils contribuent à fabriquer ce que seront nos humanités à venir. Dès lors que ces facteurs assez particuliers accomplissent au mieux leur tâche à partir d'une position de repli envers les flots de courrier quotidien, ceux d'entre nous dont nous célébrons aujourd'hui la retraite seront donc en meilleure position que jamais pour poursuivre et intensifier encore leur travail de facteurs d'humanités.

Yves Citton

Université de Grenoble Alpes

UMR LIRE (cnrs 5611) 


\section{BIBLIOGRAPHIE}

Apter, Emily, The translation zone. A new comparative literature, Princeton, Princeton University Press, 2005.

—, "Pour une politique de la traduction", La Revue des Livres, 2 (2011), p. 44-52.

Barthes, Roland, La préparation du roman. Cours et séminaires du Collège de France (1978-1980), Paris, Seuil, 2003.

Cassin, Barbara, "Présentation", in Vocabulaire européen des philosophies. Dictionnaire des intraduisibles, dir. par Barbara Cassin, Paris, Seuil, 2004, p. XVII-XXII.

Citton, Yves, Lire, interpréter, actualiser. Pourquoi les études littéraires?, Paris, Editions Amsterdam, 2007.

—, L'avenir des humanités. Economie de la connaissance ou cultures de l'interprétation?, Paris, La Découverte, 2010.

-, Gestes d'humanités. Anthropologie sauvage de nos expériences esthétiques, Paris, Armand Colin, 2012.

Felski, Rita, "Context Stinks!», New Literary History, 42 (2011), p. 573-591.

Giglioli, Daniele, "Trois cercles: critique et théorie entre crise et espoirs", La Revue des Livres, 6 (2012), p. 60-65.

Glissant, Edouard, Poétique de la relation, Paris, Gallimard, 1990.

Goma Lanzon, Javier, Ejemplaridad publica, Madrid, Taurus, 2009.

Hampton, Timothy, Writing from History. The rhetoric of exemplarity in Renaissance literature, Ithaca, Cornell University Press, 1990.

Hiвou, Béatrice, La bureaucratisation du monde à l'ère néolibérale, Paris, La Découverte, 2012.

Kelly, Christopher, Rousseau's exemplary life: the "Confessions" as political philosophy, Ithaca, Cornell University Press, 1987.

Macé, Marielle, Façons de lire, manières d'être, Paris, Gallimard, 2011.

MÉNIL, Alain, Les voies de la créolisation. Essai sur Edouard Glissant, Grenoble, De l'incidence, 2011. 
ReICHLeR, Claude, "La littérature comme interprétation symbolique», in L'interprétation des textes, dir. par Claude Reichler, Paris, Editions de Minuit, 1989, p. 81-113.

Renken, Arno, Babel heureuse - Pour lire la traduction, Paris, Van Dieren Editeur, 2012.

Rousseau, Jean-Jacques, Discours sur l'origine des langues, Paris, Gallimard, 1990 (Folio).

Shusterman, Richard, Sous l'interprétation, Paris, Editions de l'Eclat, 1994.

STIEgler, Bernard, Economie de l'hypermatériel et psychopouvoir, entretiens avec Philippe Petit et Vincent Bontemps, Paris, Mille et une nuits, 2008.

STIEgler, Bernard et al., Réenchanter le monde: la valeur esprit contre le populisme industriel, Paris, Flammarion, 2006 (Champs).

Viveiros de Castro, Eduardo, Métaphysiques cannibales, Paris, PUF, 2009.

Whitehead, Alfred North, Procès et réalité, Paris, Gallimard, 1995 (1929).

Wyss, André, Jean-Jacques Rousseau. L'accent de l'écriture, Neuchâtel, La Baconnière, 1988.

—, Eloge du phrasé, Paris, PUF, 1999. 